\title{
AVALIAÇÃO DO EFEITO DA COLOSTOMIA PROXIMAL NA CICATRIZAÇÃO DE ANASTOMOSES COLOCÓLICAS EM RATOS COM OBSTRUÇÃO INTESTINAL
}

\author{
EFFECTS OF PROXIMAL COLOSTOMY ON THE HEALING \\ OF COLONIC ANASTOMOSIS IN RATS WITH INTESTINAL \\ OBSTRUCTION
}

\author{
Marcelo Betim Paes Leme, TCBC-RJ ${ }^{1}$ \\ Delcio Matos ${ }^{2}$ \\ Loreley Andrade Luderer ${ }^{3}$ \\ Denise Arêdes Fonseca ${ }^{4}$
}

\begin{abstract}
RESUMO: Objetivo: Avaliar o efeito da colostomia proximal na cicatrização de anastomoses colocólicas em ratos com obstrução intestinal. Método: 72 ratos foram divididos em três grupos: grupo controle (C), submetido à anastomose colocólica e à colostomia proximal na ausência de oclusão intestinal; grupo sem colostomia (SC), submetido à oclusão intestinal de 72 horas e à anastomose colocólica primária; grupo com colostomia (CC) submetido à oclusão intestinal de 72 horas, à anastomose colocólica primária e à colostomia proximal. A cicatrização anastomótica foi avaliada em dois períodos, nos $2^{\circ}$ e $7^{\circ}$ dias de pós-operatório, em relação à deiscência anastomótica, aderências, epitelização mucosa, pressão de ruptura e a variáveis histológicas por estudo convencional e informatizado. Resultados: verificou-se maior tendência a deiscência anastomótica no grupo SC (12,5\%), e elevada incidência de complicações da colostomia no grupo CC (13\%), entretanto tais resultados não apresentaram diferença estatística significante. No que se refere às demais variáveis analisadas para verificação da cicatrização anastomótica deve-se considerar que houve equivalência entre os três grupos nos dois períodos analisados. Conclusão: Não há diferença entre a cicatrização de anastomoses colocólicas associadas ou não à colostomia proximal, em ratos com obstrução intestinal.
\end{abstract}

Descritores: Colostomia; Obstrução intestinal; Cólon.

\section{INTRODUÇÃO}

O tratamento da obstrução cólica esquerda ainda permanece um assunto controverso. Uma das principais questões refere-se à realização ou não de uma anastomose primária no colo obstruído. Nota-se que existe atualmente uma preferência pela realização da anastomose primária ${ }^{1-3}$, entretanto, muitas questões relativas aos riscos e benefí- cios nessa situação ainda não foram satisfatoriamente respondidas. Principalmente quando se verifica, que foram relatadas taxas elevadas de deiscência, em anastomoses realizadas em vigência de oclusão intestinal ${ }^{4-7}$.

O uso da colostomia proximal como proteção das anastomoses colocólicas e colorretais, especialmente naquelas consideradas de risco, tem sido sugerido por alguns autores $^{8-10}$. Outros autores, no entanto, acreditam que a

1. Professor Assistente da Disciplina de Clínica Cirúrgica do Curso de Medicina do Centro Universitário de Volta Redonda - UniFOA. Mestre em Gastroenterologia Cirúrgica pela UNIFESP-EPM.

2. Professor Livre Docente e Chefe do Grupo de Coloproctologia da Disciplina de Gastroenterologia Cirúrgica da UNIFESP-EPM.

3. Professora Titular da Disciplina de Patologia do Curso de Odontologia do Centro Universitário de Volta Redonda UniFOA.

4. Acadêmica do 5ำ ano do Curso de Medicina do Centro Universitário de Volta Redonda - UniFOA.

Recebido em 12/7/2000

Aceito para publicação em 21/11/2000

Pesquisa elaborada no Curso de Pós-Graduação da Disciplina de Gastroenterologia Cirúrgica da Escola Paulista de Medicina - UNIFESP-EPM, Desenvolvida no Laboratório de Cirurgia Experimental do Curso de Medicina do Centro Universitário de Volta Redonda - UniFOA. 
presença da colostomia proximal não evita ou diminui as taxas de deiscência anastomótica ${ }^{11,12}$.

Tem-se considerado como vantagem ao uso das colostomias o melhor controle das complicações sépticas originadas da deiscência anastomótica ${ }^{11,12}$ e como desvantagem as taxas significativas de morbidade e mortalidade atribuídas a este procedimento ${ }^{13-16}$.

Portanto, a falta de evidência científica conclusiva, se em vigência de obstrução intestinal a realização da colostomia proximal serve como fator de proteção para a anastomose, nos motivou à realização desta pesquisa que teve como objetivo avaliar o efeito da colostomia proximal na cicatrização de anastomoses colocólicas em ratos com obstrução intestinal.

\section{MÉTODO}

Esta pesquisa foi idealizada no programa de Pós-Graduação da Disciplina de Gastroenterologia Cirúrgica, da UNIFESP - Escola Paulista de Medicina, aprovada pela Comissão de Ética do HSP/UNIFESP e desenvolvida no Laboratório de Cirurgia Experimental do Curso de Medicina do Centro Universitário de Volta Redonda - UniFOA.

Foram utilizadas no experimento 72 ratas (Rattus Norvegicus Albinus), da linhagem Wistar, com idade em torno de 120 dias e peso médio de 227 gramas, divididas aleatoriamente em três grupos de 24 animais: grupo controle (C), grupo sem colostomia (SC) e grupo com colostomia (CC).

O tamanho da amostra foi calculado considerandose um erro Alfa $<5 \%$, erro Beta $=20 \%$ e uma diferença esperada entre os grupos para a principal variável - deiscência anastomótica - de 30\%. Baseado no estudo-piloto considerou-se ainda uma perda de $10 \%$, resultando então no número de 24 animais por grupo.

$\mathrm{O}$ grupo $\mathrm{C}$ foi submetido, no primeiro tempo, à laparotomia mediana e num segundo tempo (72 horas após) à ressecção cólica segmentar seguida de anastomose colocólica primária e complementada com uma colostomia proximal.

Os grupos $\mathrm{SC}$ e CC foram submetidos, no primeiro tempo, à oclusão intestinal de 72 horas e num segundo tempo à ressecção cólica envolvendo a área da obstrução seguida de anastomose colocólica primária. Os animais do grupo CC foram destinados à colostomia proximal complementar, e os do grupo SC não foram submetidos a nenhum procedimento complementar.

Os ratos receberam anestesia inalatória, com éter sulfúrico. $\mathrm{O}$ acesso à cavidade abdominal foi realizado através de uma laparotomia mediana de $5 \mathrm{~cm}$ de extensão.

Nos grupos SC e CC a oclusão intestinal foi realizada utilizando-se a introdução de um cateter nasogástrico número quatro por via retal, que foi posicionado no colo esquerdo. Posteriormente, por abertura feita no mesocolo a $2,5 \mathrm{~cm}$ da reflexão peritoneal, foi passado um fio de algodão 000, e realizada uma ligadura sobre tubo previamente posicionado, evitando-se desta forma o sofrimento vascular. ${ }^{17}$ Nos animais do grupo C, submetidos no primeiro tempo à laparotomia mediana, foram respeitados os mesmos tempos operatórios, exceto pela passagem e fixação do fio sobre o colo.

No segundo tempo operatório, 72 horas após a primeira intervenção, foi realizada a ressecção segmentar do cólon esquerdo de um centimetro de extensão interessando a zona de oclusão, grupos SC e CC, ou o sítio anatômico correspondente nos animais do grupo C, submetidos anteriormente à laparotomia mediana.

A reconstrução do trânsito foi feita através de anastomose término-terminal, em plano único de sutura seromuscular (extramucoso) com 8 a 10 pontos separados, confeccionados com fio de polipropileno 6-0 com agulha cilíndrica. Utilizou-se o auxílio de uma lupa cirúrgica, marca Intex (Ref. 28 X 32 LP), que proporcionou um aumento de $4 x$.

A colostomia proximal foi realizada nos grupos $\mathrm{C}$ e CC, pela secção do colo, na flexura cólica esquerda e exteriorização da boca proximal, no quadrante superior esquerdo do abdome. A alça distal foi fechada por sutura contínua e deixada na cavidade peritoneal.

A morte dos animais, para estudo das anastomoses, foi realizada com dose inalatória letal de éter. Doze de cada grupo foram mortos no segundo e sétimo dias de pós-operatório, correspondente à realização da anastomose, constituindo-se assim dois subgrupos de 12 animais.

Os ratos que morreram antes do período determinado foram submetidos à laparotomia, com finalidade de verificar se a causa do óbito estava relacionada a complicações da operação e, particularmente, à deiscência anastomótica ou relacionada à colostomia.

A deiscência anastomótica foi considerada presente quando se verificava os achados de abscesso perianastomótico, peritonite difusa e da própria deiscência. As aderências foram classificadas como ausentes, ou se presentes, de acordo com número de quadrantes que ocupavam.

Após a avaliação macroscópica da cavidade, foi retirado um segmento de $4 \mathrm{~cm}$ do colo esquerdo contendo a anastomose centralizada para avaliação da sua resistência e verificação da epitelização da ferida mucosa.

Para realização do teste de resistência o espécime cirúrgico foi imerso em cuba contendo água e o ar insuflado de 10 em $10 \mathrm{mmHg}$, a cada 15 segundos, até que se percebesse o borbulhar de ar na água evidenciando a ruptura da peça $^{17,18}$. As pressões foram registradas em manômetro graduado em mmHg e conectado em Y ao sistema. Foram excluídas deste teste as anastomoses que apresentaram sinais macroscópicos de deiscência.

Posteriormente, a peça foi aberta pelo bordo antimesenterial, através de uma incisão longitudinal. A área interna da anastomose foi inspecionada e avaliada a epitelização mucosa da zona anastomótica, que foi classificada considerando-se o número de terços epitelizados na linha anastomótica.

Dois fragmentos da zona anastomótica, um correspondente à borda mesenterial e outro à antimesenterial da alça intestinal, foram encaminhados para estudo histológico em coloração pela hematoxilina-eosina.

As variáveis necrose, inflamação aguda, inflamação crônica, regeneração mucosa, fibrose e tecido de granula- 
ção foram pesquisadas à microscopia óptica, na região cicatricial, quanto a sua presença e intensidade. Foi estabelecido o seguinte critério para quantificação das alterações: $0=$ ausência de alteração, $1=$ alterações presentes em grau leve, 2 = alterações presentes em grau moderado e $3=$ alterações presentes em grau intenso.

O examinador das lâminas histológicas não estava informado a respeito da origem dos espécimes, se de anastomoses do grupo $\mathrm{C}, \mathrm{SC}$ ou $\mathrm{CC}$.

No estudo histológico informatizado as imagens obtidas das anastomoses foram digitalizadas e analisadas no Laboratório de Informática Dedicado à Odontologia, da Disciplina de Patologia Geral da Faculdade de Odontologia da Universidade de São Paulo, através do programa DIRACOM 3 de análise gráfica.

Pela subtração de imagens foi possível analisar quantitativamente três variáveis do processo cicatricial anastomótico. A celularidade, determinada pela aferição da nuclearidade; o edema, determinado pela quantificação dos espaços intercelulares; a matriz protéica, determinada pela exclusão das duas anteriores. Os resultados foram apresentados sob forma do percentual existente de cada uma destas variáveis nos campos examinados.

$\mathrm{Na}$ avaliação dos resultados encontrados foram aplicados testes estatísticos com a finalidade de se verificar a significância das diferenças encontradas. Foram utilizados os seguintes testes: intervalo de confiança de 95\%, Kruskal-
Wallis, Qui-quadrado, exato de Fisher (bicaudal) e MannWhitney. Considerou-se como significativos valores de $\mathrm{p}<0,05$.

\section{RESULTADOS}

Durante o experimento foram registrados sete óbitos, $9,7 \%$ do total de animais: três por complicações da colostomia, três em decorrência da anestesia e um por deiscência anastomótica. Em relação à deiscência anastomótica foi possível observar que esta ocorreu somente no grupo SC. A frequiência de deiscência anastomótica neste grupo foi de $12,5 \%$. Verificou-se também que as complicações da colostomia ocorreram somente no grupo CC com uma freqüência de $13 \%$ (Tabela 1).

As aderências perianastomóticas estiveram presentes em todos os grupos nos dois períodos analisados. No $2^{\circ}$ dia verificou-se uma menor intensidade de aderências no grupo $\mathrm{C}$ quando comparado aos grupos SC e CC. Essa diferença foi estatisticamente significante $(p<0,001)$. No 7o dia nãose observou diferença significativa na freqüência e intensidade das aderências perianastomóticas entre os três grupos (Tabela 2).

Em relação aos níveis de pressão de ruptura não foi possível se detectar diferença entre os três grupos nos dois períodos estudados, 2 e 7o dia (Tabela 3). Quando se comparou as pressões registradas no $2^{\circ}$ dia com as pressões

Tabela 1

Frequiência de deiscência anastomótica e complicações da colostomia

\begin{tabular}{|c|c|c|c|c|}
\hline Eventos & Grupo C (\%) & Grupo SC (\%) & Grupo CC (\%) & $p$ \\
\hline Deiscência & 0,0 & 12,5 & 0,0 & $\geq 0,05$ \\
\hline Complicações da colostomia & 0,0 & 0,0 & 13,0 & $\geq 0,05$ \\
\hline
\end{tabular}

Tabela 2

Frequiência de aderências perianastomóticas ocupando 3 ou 4 quadrantes

\begin{tabular}{|c|c|c|c|}
\hline Número de quadrantes & Grupo C (\%) & Grupo SC (\%) & Grupo CC (\%) \\
\hline $2^{\circ} \mathrm{PO}^{*}$ & 41,7 & 100,0 & 100,0 \\
\hline 7ㅇ $\mathrm{PO}$ & 70,0 & 100,0 & 90,0 \\
\hline
\end{tabular}

"Grupo C" $\neq$ "Grupo CC" = "Grupo SC".

* Fischer (bicaudal): “Grupo C” versus "Grupos $S C+C C$ ” $p<0,001$.

Tabela 3

Freqüência de rupturas no espécime operatório, segundo os níveis de pressão em mmHg

\begin{tabular}{|c|c|c|c|c|c|}
\hline Sacrificio & $\begin{array}{l}\text { Pressão de ruptura } \\
(\mathrm{mm} \mathrm{Hg})\end{array}$ & Grupo C (\%) & Grupo SC (\%) & Grupo CC (\%) & $p$ \\
\hline 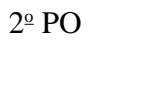 & $\begin{array}{l}\text { Até } 60 \\
61 \text { a } 180\end{array}$ & $\begin{array}{l}25,0 \\
75,0\end{array}$ & $\begin{array}{l}40,0 \\
60,0\end{array}$ & $\begin{array}{l}40,0 \\
60,0\end{array}$ & $\begin{array}{l}\geq 0,05 \\
\geq 0,05\end{array}$ \\
\hline 7 으 $\mathrm{PO}$ & $\begin{array}{c}61 \text { a } 180 \\
181 \text { a } 300\end{array}$ & $\begin{array}{l}20,0 \\
80,0\end{array}$ & $\begin{array}{l}36,4 \\
63,6\end{array}$ & $\begin{array}{l}20,0 \\
80,0\end{array}$ & $\begin{array}{l}\geq 0,05 \\
\geq 0,05\end{array}$ \\
\hline
\end{tabular}


registradas no 70 dia, nos três grupos, verificou-se que as pressões no $2^{\circ}$ dia foram sistematicamente mais baixas do que no 7 o dia. Essa diferença foi estatisticamente significante $(\mathrm{p}<0,001)$.

Pôde-se verificar ainda que no $2^{\circ}$ dia as rupturas ocorreram com maior freqüência na anastomose e que no 7o dia ocorreram com maior freqüência na alça intestinal (Gráfico 1).

Em relação à epitelização mucosa observou-se que a freqüência de cobertura epitelial na linha anastomótica $>$ $2 / 3$, foi menos freqüente no grupo SC tanto no 2 o quanto no 7o dia de pós-operatório (PO.). Entretanto as diferenças não foram significativas (Tabela 4).

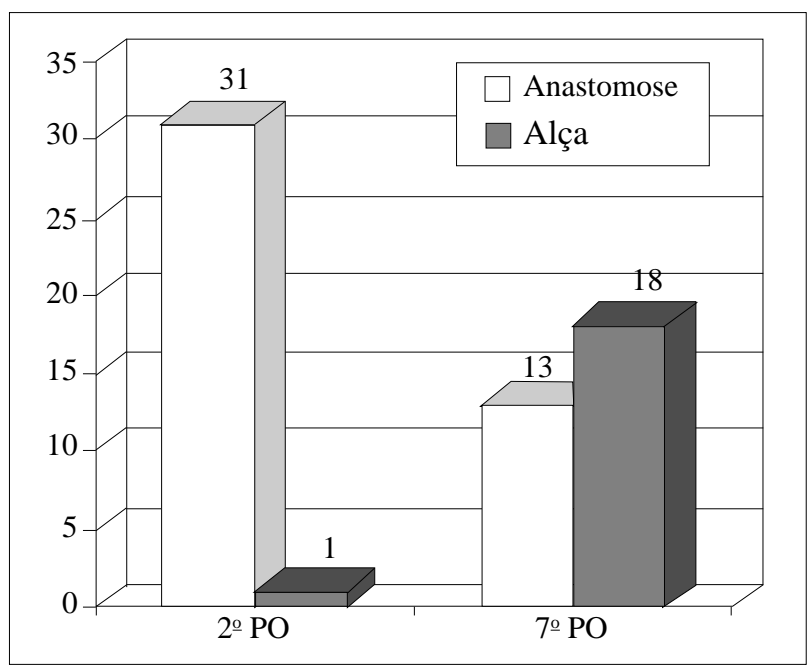

Gráfico 1 - Local de ruptura - Comparação entre o $2^{\circ}$ e $7^{\circ} \mathrm{PO}$. Qui-quadradado $p<0,001$.
O estudo histológico convencional revelou equivalência, na maioria das variáveis analisadas, entre os três grupos estudados, tanto no 2 o quanto no 7으. POrificouse que a necrose e a inflamação aguda estiveram presentes com maior intensidade no $2^{\circ}$ dia e que a inflamação crônica, a fibrose e o tecido de granulação estiveram presentes com maior intensidade no $7^{\circ} \mathrm{o}$ dia.

No estudo histológico informatizado verificou-se que a nuclearidade, a matriz protéica e os interstícios foram equivalentes nos três grupos tanto no $2^{\circ}$ quanto no 7o dia (Tabela 5). Quando se comparou o 2o dia com o 7o dia de pós-operatório verificou-se que o edema foi maior no $2^{\mathrm{o}}$ dia em todos os grupos. Essa diferença foi significante $(\mathrm{p}<0,001)$.

\section{DISCUSSÃO}

A deiscência anastomótica é considerada a principal complicação das operações sobre o intestino grosso e deve servir como principal parâmetro na avaliação de uma anastomose. Em estudos clínicos, a sua freqüência, na vigência de oclusão intestinal, é muito variável e repetidamente elevada. 4, 5, 6,7 Experimentalmente também se verifica uma elevada incidência de deiscências nas anastomoses realizadas em colo obstruído. 19, 20, 21

A influência da colostomia proximal sobre as anastomoses colocólicas, em colo não obstruído, no que se refere à incidência da deiscência anastomótica, foi estudada por diversos autores e os resultados são conflitantes. Assim, Blomquist et al. ${ }^{22}$ e Udén et al. ${ }^{23}$ observaram maior incidência de deiscência nas anastomoses sem colostomia proximal; Senagore et al. ${ }^{24}$ não detectaram diferenças significativas entre o grupo com e sem colostomia proximal e Bielecki et al. ${ }^{25}$ verificaram maior freqüência de deiscências radiológicas nas anastomoses do grupo com colostomia.

Tabela 4

Frequiência de anastomoses com epitelização mucosa maior do que 2/3

\begin{tabular}{c|c|c|c|c}
\hline Dia da morte & Grupo C (\%) & Grupo SC (\%) & Grupo CC $(\%)$ & $p$ \\
\hline 2o PO & 83,3 & 16,7 & 40,0 & $\geq 0,05$ \\
70 PO & 70,0 & 18,2 & 60,0 & $\geq 0,05$ \\
\hline
\end{tabular}

Tabela5

Percentual de achados histológicos no estudo informatizado

\begin{tabular}{l|c|c|c|c}
\hline Sacrifício & Eventos & Grupo C (\%) & Grupo SC (\%) & Grupo CC(\%) \\
\hline \multirow{2}{2}{ o PO } & Nuclearidade & 9,66 & 9,64 & 10,40 \\
& M. Protéica & 61,18 & 59,59 & 62,60 \\
& Interstícios & 29,17 & 30,77 & 27,00 \\
\hline 7 PO & Nuclearidade & 10,76 & 12,94 & 9,56 \\
& M. Protéica & 66,68 & 64,18 & 65,46 \\
& Interstícios & 22,61 & 22,88 & 24,98 \\
\end{tabular}

Mann-Whitney - porcentagem de interstícios no $2^{\circ}$ PO versus $7^{\circ} \mathrm{PO} p<0,001$. 
Entretanto, o efeito da colostomia proximal sobre as anastomoses colocólicas, realizadas na vigência de obstrução intestinal, não tem sido objeto de muitos estudos. Nas bases de dados pesquisadas só foi possível encontrar uma única publicação, feita por Törnqvist et al. ${ }^{21}$, que detectou uma maior freqüência de deiscência nas anastomoses colocólicas não associadas à colostomia proximal.

No presente experimento não se verificou diferença estatisticamente significante na incidência de deiscência anastomótica entre os diversos grupos. Contudo, quando se comparou a incidência de deiscência anastomótica no grupo SC, onde esta ocorreu em 12,5\% animais, com o grupo CC no qual nenhuma deiscência foi registrada, podese questionar um possível efeito protetor da colostomia proximal sobre as anastomoses colocólicas realizadas em colo obstruído.

As aderências perianastomóticas estiveram presentes em todos os grupos nos dois períodos analisados. A grande freqüência de aderências perianastomóticas, também foi observada por outros autores que estudaram as anastomoses cólicas ${ }^{17,26}$.

Nessa pesquisa, observou-se que nas anastomoses realizadas em colo obstruído, a realização de uma colostomia proximal não foi suficiente para produzir diferenças significativas, tanto na intensidade quanto na freqüência, das aderências perianastomóticas. Entretanto verificou-se, no 2 o dia, uma intensidade significativamente menor de aderências perianastomóticas no grupo não ocluído.

Uma possível explicação para a maior intensidade de aderências perianastomóticas nos grupos com oclusão intestinal, como verificado nesta pesquisa, poderia ser a presença de fatores que estimulassem a formação de aderências, tais como a presença de uma maior carga fecal intraluminal ${ }^{27}$ ou a reação inflamatória que ocorre na parede do colo obstruído. ${ }^{28}$

O teste de resistência das anastomoses não demonstrou diferença significativa na pressão de ruptura entre os diversos grupos. Contudo, quando se comparou as pressões de ruptura no $2^{\circ}$ dia com as observadas no 70 dia as primeiras foram sistematicamente mais baixas, resultando em diferenças significativas. Tal ocorrência tem sido repetidamente observada na literatura quando se compara a fase inicial da cicatrização, quando a resistência da anastomose é mais dependente das suturas, com as fases mais tardias onde a reparação tecidual e a deposição de colágeno estão presentes de forma mais definitiva ${ }^{18,29,30,31}$.

Alguns autores verificaram ainda, que a resistência da anastomose na segunda fase da cicatrização tende a ser igual ou superior à imposta pela parede intestinal e que, portanto, um maior percentual de rupturas deveria teoricamente ocorrer fora do sítio da anastomose $29,32,33$

Nesta pesquisa verificou-se que no $2^{\circ}$ dia as rupturas ocorreram com maior frequiência nas anastomoses e que no 7을 dia ocorreram mais freqüentemente na alça intestinal. Verificou-se também que, no 7o dia, o grupo com colostomia (CC) apresentou um maior número de rupturas na anastomose $(60 \%)$ e que o grupo sem colostomia (SC) apresentou um maior número de rupturas na alça intestinal $(63,3 \%)$. Embora essas diferenças não tenham demonstra- do significância estatística, a possibilidade de que a colostomia proximal tenha o efeito de diminuir a resistência anastomótica, como previamente sugerido por Blomquist et al. ${ }^{22}$, não pode ser afastada.

A análise da face mucosa das anastomoses demonstrou uma tendência de menor grau de epitelização no grupo sem colostomia. É possível que o maior contato e trânsito do conteúdo fecal na luz intestinal e, portanto, na linha anastomótica tenham contribuído para formação de úlceras mucosas nestas anastomoses. Tal diferença entretanto não atingiu valores de significância estatística.

A análise estatística dos eventos histológicos entre os três grupos revelou equivalência na maioria dos itens estudados. Koruda \& Rolandelli ${ }^{34}$ e Hendriks \& Mastboom $^{31}$ relataram que esta semelhança nos achados histológicos se deve principalmente à seqüência natural de eventos que ocorrem de forma muito semelhante no processo de regeneração tecidual de qualquer anastomose intestinal.

$\mathrm{Na}$ tentativa de se quantificar de forma mais exata os fenômenos histológicos da cicatrização anastomótica, três variáveis foram analisadas pelo estudo histológico informatizado. Entre outros recursos o programa permite que seja selecionada determinada estrutura, tendo como base sua cor e então calculada a área percentual que esta estrutura representa no campo examinado. Foram analisados por este método a nuclearidade, a presença relativa de infiltrado intersticial (edema) e a matriz protéica, e não foram observadas diferenças significativas entre os diversos grupos.

Pode-se verificar entretanto que na fase inicial da cicatrização, $2^{\mathrm{o}}$ dia, havia uma intensidade significativamente maior de espaço intersticial, edema, que as observadas no 7o dia. Tal achado, apesar de largamente comentado na literatura, sempre foi analisado de maneira empírica. O presente trabalho, através do método de estudo histológico informatizado, permitiu quantificar, de forma mais precisa, o edema anastomótico. Estas mesmas conclusões sobre a eficácia do método na quantificação do edema anastomótico foram observadas por outros autores ${ }^{35,36}$.

A anestesia foi responsável por três óbitos ocorridos no experimento. A oclusão intestinal parece não ter influenciado na mortalidade anestésica já que dois óbitos ocorreram no grupo sem oclusão e um no grupo com oclusão. A deiscência anastomótica, embora presente em três animais do grupo SC, só foi responsável pelo óbito de um deles.

A colostomia foi responsável por três óbitos ocorridos no experimento, perfazendo uma taxa de $6,7 \%$ quando se considera os animais dos dois grupos submetidos a este procedimento ( $\mathrm{C}$ e $\mathrm{CC}$ ). Entretanto quando se verifica que todos os óbitos decorrentes de complicações da colostomia ocorreram no grupo com obstrução intestinal, nota-se que a mortalidade neste grupo foi de $13 \%$.

Parece portanto pertinente considerar a possibilidade de que a oclusão intestinal seja um dos fatores que contribuem para este desfecho. A colostomia é sabidamente um procedimento operatório com índices de complicação não 
desprezíveis. ${ }^{15,37}$ Contudo não foi possível verificar na literatura pesquisada se a situação de obstrução intestinal modificaria as taxas de complicação deste procedimento, como observado nesse experimento.

Podem-se questionar como fatores que influenciaram nestes resultados o maior calibre da alça intestinal, uma possível isquemia relativa da parede intestinal e maior carga fecal da víscera.
Com base nos resultados obtidos na presente pesquisa, podemos concluir que: não há diferença entre a cicatrização de anastomoses colocólicas associadas ou não à colostomia proximal, em ratos com oclusão intestinal.

Assim, é preciso questionar se o possível benefício de uma colostomia proximal, em diminuir a frequiência das deiscências anastomóticas, não poderia estar comprometido pela incidência de complicações próprias da colostomia.

\begin{abstract}
Background: Our objective is to evaluate the effects of the proximal colostomy on the healing of colonic anastomosis in rats with intestinal obstruction. Method: 72 rats were allocated into three goups: control group $(C)$ with no intestinal occlusion, was subjected to colonic anastomosis and proximal colostomy; non-colostomy group (SC) with 72 hours left colon obstruction under went a colonic ressection and primary anastomosis; colostomy group (CC) with a 72 hours left colon obstruction of and a colonic ressection, primary anastomosis and proximal colostomy. Anastomosis healing was evaluated by the presence of anastomotic leaking and mucosal line, adhesions, bursting presure, conventional and computerized histologic examination at the $2^{\text {nd }}$ and $7^{\text {th }}$ potoperative days. Results: The incidence of anastomotic leaking was 12,5\% in group SC while in group CC there was an incidence of colostomy complications in 13\%, with no significant statistical difference. No difference was noticied in the other healing anastomotic variables. Conclusions: There is no difference in the healing of colonic anastomosis related to the presence or abscence of a proximal colostomy, in rats with intestinal obstruction.
\end{abstract}

Key Words: Colostomy; Intestinal obstruction; Colon.

\section{REFERÊNCIAS}

1. Almeida ACM, Gracias CW, Santos MN, et al. - Abordagem cirúrgica da obstrução maligna aguda do cólon esquerdo: o declínio da colostomia. Rev Bras ColoProct 1991, 11:48-54.

2. Silva JS, Kerzner A, Formiga GJS, et al. - Lavagem intestinal anterógrada transoperatória na obstrução neoplásica do cólon esquerdo. Rev Bras Colo-Proct 1993, 13:42-5.

3. Ross S, Krukowski ZH, Munro A, et al. - Single-stage treatment for left-sided colonic obstruction: a prospective randomized clinical trial comparing subtotal colectomy with segmental resection following intraoperative irrigation. Br J Surg 1995, 82:1622-27.

4. Irvin T \& Goligher JC - Aetiology of disruption of intestinal anastomose. Brit J Surg 1973, 60:461-4.

5. Fielding LP \& Wells BW - Survival after primary and after staged resection for large bowel obstruction caused by cancer. Br J Surg 1974, 61:16-1.

6. Irvin TT \& Greaney G - The treatment of colonic cancer presenting with intestinal obstruction. Br J Surg 1977, 64:741-4

7. Phillips RKS, Hittinger R, Fry JS, et al. - Malignant large bowel obstruction. Br J Surg 1985, 72:296-302.

8. McSherry CK, Grafe WR, Perry HS, et al. - Surgery of the large bowel for emergent conditions. Arch Surg 1969, 98:749-53.

9. Goligher JC, Graham NG, Dombal FT - Anastomotic dehiscence after anterior resection of rectum and sigmoid. Br J Surg 1970, 57:109-18.

10. Valerio D \& Jones PF - Immediate resection in treatment of large bowel emergencies. Br J Surg 1978, 65:712-16.
11. Karanjia ND, Corder AP, Holdsworth PJ, et al. - Risk of peritonitis and fatal septicaemia and the need to defunction the low anastomosis. Br J Surg 1991, 78:196-8.

12. Pakkastie TE, Ovaska JT, Pekkala ES, et al. - A randomized study of colostomies in low colorectal anastomoses. Eur J Surg 1997, 163: 929-33.

13. Hopkins JE. Transverse colostomy in the management of cancer of the colon. Dis Colon Rectum 1971, 14:232-6.

14. Wara P, Sørensen K, Berg V - Proximal fecal diversion: review of ten years experience. Dis Colon Rectum 1981, 24:114-19.

15. Winkler MJ \& Volpe PA - Loop transverse colostomy: the case against. Dis Colon Rectum 1982, 25:321-26.

16. Porter JA, Salvati EP, Rubin RJ, et al. - Complications of colostomies. Dis Colon Rectum 1989, 32:299-303.

17. Biondo-Simões MLP - Estudo comparativo da evolução de anastomoses do cólon esquerdo realizadas na vigência e na ausência de obstrução aguda, com e sem limpeza, no rato. São Paulo,1994. [Tese - doutorado Escola Paulista de Medicina]

18. Cronin K, Jakson DS, Dumphy JE - Changing bursting strength and collagen content of the healing colon. Surg Gynecol Obstet 1968, 126:747-53.

19. Leaper DJ - Angiography as an index of healing in experimental laparotomy wounds and colonic anastomoses. Ann R Coll Sur Engl 1983, 65:20-3.

20. Törnqvist A, Blomquist P, Jiborn H, et al. - Anastomotic healing after resection of left-colon stenosis: effect on collagen metabolism and anastomotic strength. Dis Colon Rectum 1990, 33:217-21. 
21. Törnqvist A, Blomquist $\mathrm{P}$, Jiborn $\mathrm{H}$, et al. - The effect of diverting colostomy on anastomotic healing after resection of left colon obstruction: an experimental study in the rat. Int J Colorectal Dis 1990, 5:167-9.

22. Blomquist P, Jiborn H, Zederfeld B - Effect of diverting colostomy on breaking strength of anastomoses after resection of left side of the colon. Am J Surg 1985, 149:712-5.

23. Udén $\mathrm{P}$, Blomquist $\mathrm{P}$, Jiborn $\mathrm{H}$, et al. - Influence of proximal colostomy on the healing of a left colon anastomosis: an experimental study in rat. Br J Surg 1988, 75:32529.

24. Senagore A, Milsom JW, Walshaw RK, et al. - Does a proximal colostomy affect colorectal anastomotic healing? Dis Colon Rectum 1992, 35:182-5.

25. Bielecki K, Grotowski M, Kalczac M - Influence of proximal end diverting colostomy on the healing of left side colonic anastomosis: an experimental study in rats. Int J Colorectal Dis 1995, 10:193-6.

26. Aguilar-Nascimento JE, Caporossi C, Figueiredo PC, et al. - Anastomose primária em plano único na obstrução aguda do cólon esquerdo: estudo em ratos. Rev Bras Colo-Proct 1990, 10:143-7.

27. Blomquist $\mathrm{P}$, Jiborn $\mathrm{H}$, Zederfeld B - The effect of relative bowel rest on healing of colonic anastomoses. Acta Chir. Scand 1984, 150:671-5.

28. Törnqvist A, Forsgren A, Fäldt K, et al. - Bacterial load and inflamatory reaction in the bowel wall after colonic obstruction. Eur J Surg 1991, 157:539-542.

29. Herrmann JB, Woodward SC, Pulaski EJ - Healing of colonic anastomoses in the rat. Surg Gynecol Obstet 1964, 119:269-75.

30. Ballantyne GH - Intestinal suturing: review of the experimental foundations for tradicional doctrines. Dis Colon Rectum 1983, 26:836-43.

31. Hendriks T \& Mastboom WB - Healing of experimental intestinal anastomoses. Parameters for repair. Dis Colon Rectum 1990, 33:.891-901.

32. Jiborn $\mathrm{H}$, Ahonen J, Zederfeldt B - Healing of experimental colonic anastomoses: I. Bursting strength of the colon after left colon resection and anastomosis. Am J Surg 1978, 136:587-94.

33. Aguilar-Nascimneto JE, Mathie RT, Man WK, et al. - Enhanced intra-anastomotic healing by operative lavage with nutrient solutions in experimental left-sided colonic obstrution. Br J Surg 1995, 82:461-4.
34. Koruda MJ \& Rolandelli RH - Experimental studies on the healing of colonic anastomoses. J Surg. Res 1990, 48:.504-15.

35. Matos D, Saad SS, Franceschi O, et al. - Estudo experimental comparativo entre anastomose colocólica com sutura manual e com anel biofragmentável. Rev Ass Med Bras 1993, 39: 201-6.

36. Novelli MG, Barreto E, Matos D, et al. - Aplicação do processamento de imagens por computador na quantificação das variáveis histopatológicas da reparação tecidual de anastomoses colocólicas em ratos. Rev Ass Med Bras 1997, 43:277-82.

37. Segreti EM, Levenback C, Morris M, et al. - A comparasion of end loop colostomy for fecal diversion in gynecologic patients with colonic fistulas. Gynecol Oncol 1996; 60:49-53.

Endereço para correspondência:

Dr. Marcelo Betim Paes Leme

Rua Moacir de Paula Lobo, 19

27283-350 — Volta Redonda-RJ

e-mail: marcelo.leme@uol.com.br 